\title{
Effet de la fertilisation organique des étangs à partir des fientes de poulets sur la survie et la croissance des alevins de Oreochromis niloticus (Linnaeus, 1758)
}

\author{
Amon Yao Nicolas \\ Université Peleforo GON COULIBALY, UFR-Sciences biologiques, \\ Département de Biologie Animale, Korhogo, Côte d'Ivoire \\ Coulibaly André \\ Université de MAN, UFR-Ingénierie Agronomique Forestière et \\ Environnementale, Man, Côte d'Ivoire \\ Kouakou N'Gouan Akpolët \\ Université Peleforo GON COULIBALY, UFR-Sciences biologiques, \\ Département de Biologie Animale, Korhogo, Côte d'Ivoire

\section{Yao Kouakou} \\ Université NANGUI ABROGOUA, UFR-SN, Laboratoire de Biologie et \\ Cytologie Animales, Abidjan, Côte d'Ivoire
}

Doi:10.19044/esj.2021.v17n14p246

Submitted: 08 February 2021

Accepted: 31 March 2021

Published: 30 April 2021
Copyright 2021 Author(s)

Under Creative Commons BY-NC-ND

4.0 OPEN ACCESS

Cite As:

Nicolas A.Y., André C., Akpolët K.N. \& Kouakou Y. (2021). Effet de la fertilisation organique des étangs à partir des fientes de poulets sur la survie et la croissance des alevins de Oreochromis niloticus (Linnaeus, 1758). European Scientific Journal, ESJ, 17(14), 246. https://doi.org/10.19044/esj.2021.v17n14p246

\section{Resume}

Pour apprécier l'efficacité de l'engrais organique dans la production piscicole, une étude a été réalisée sur l'effet des fientes de poulets sur la survie et la croissance des alevins de Oreochromis niloticus en phase de prégrossissement en étang. Pour ce faire, 24000 alevins de cette espèce de poids moyens compris entre $3,93 \pm 0,62$ get $3,97 \pm 0,31 \mathrm{~g}$ ont été sélectionnés et répartis équitablement dans six (6) étangs de $200 \mathrm{~m}^{2}$ à la densité de 20 alevins $/ \mathrm{m}^{2}$. Trois étangs ont reçu une application de fientes de poulet à la dose $10 \mathrm{~kg} / \mathrm{m}^{2} /$ mois tandis que les trois autres, pris comme témoins, n'ont pas été fertilisés. Les alevins ont été nourris trois fois par jour à l'aide d'un aliment farineux titrant $35 \%$ de protéines à la ration journalière de $10 \%$ de la biomasse 
durant deux mois. Les résultats relatifs aux performances de croissance indiquent que le Gain Moyen Quotidien (GMQ) a été plus important au niveau des poissons élevés dans les étangs fertilisés que celui enregistré au niveau des étangs non fertilisés $(1,04 \pm 0,17 \mathrm{~g} / \mathrm{j}$ contre $0,69 \pm 0,11 \mathrm{~g} / \mathrm{j})$. En ce qui concerne le taux de croissance spécifique pondéral, son évolution a été semblable à celui du GMQ. La valeur enregistrée a été de 4,71 $\pm 0,22 \% / j$ pour les poissons des étangs fertilisés contre $4,07 \pm 0,01 \% / \mathrm{j}$ pour les poissons des étangs non fertilisés. Quant à la survie, de l'ordre de $95 \%$, elle a été sensiblement identique dans tous les milieux d'élevage. Ces résultats suggèrent que l'apport de fertilisant (fientes) contribue à la croissance des poissons par l'augmentation de la production primaire, source supplémentaire de nourriture.

Mots clés : Fertilisation organique, croissance, survie, Oreochromis niloticus

\title{
Effect of Organic Fertilization of Ponds from Chicken Droppings on the Survival and Growth of Oreochromis Niloticus Fry (Linnaeus, 1758)
}

\author{
Amon Yao Nicolas \\ Université Peleforo GON COULIBALY, UFR-Sciences biologiques, \\ Département de Biologie Animale, Korhogo, Côte d'Ivoire \\ Coulibaly André \\ Université de MAN, UFR-Ingénierie Agronomique Forestière et \\ Environnementale, Man, Côte d'Ivoire \\ Kouakou N'Gouan Akpolët \\ Université Peleforo GON COULIBALY, UFR-Sciences biologiques, \\ Département de Biologie Animale, Korhogo, Côte d'Ivoire \\ Yao Kouakou \\ Université NANGUI ABROGOUA, UFR-SN, Laboratoire de Biologie et \\ Cytologie Animales, Abidjan, Côte d'Ivoire
}

\begin{abstract}
To assess the effectiveness of organic fertilizer in fish production, a study was carried out on the effect of chicken droppings on the survival and growth of Oreochromis niloticus fry in the first stages of their breeding in ponds. To do this, 24000 fry of this species of average weight between 3.93 $\pm 0.62 \mathrm{~g}$ and $3.97 \pm 0.31 \mathrm{~g}$ were selected and distributed equally in six (6) ponds of $200 \mathrm{~m}^{2}$ at a density of $20 \mathrm{fry} / \mathrm{m}^{2}$. Three ponds received an application of chicken droppings at a dose of $10 \mathrm{~kg} / \mathrm{m}^{2} /$ month while the other three, taken
\end{abstract}


as controls, were not fertilized. The fry were fed three times a day with a floury feed containing $35 \%$ protein in the daily ration of $10 \%$ of the biomass during two months. The results relating to growth performance indicate that the Daily Weight Gain (DWG) was greater in fish reared in fertilized ponds than that recorded in unfertilized ponds $(1.04 \pm 0.17 \mathrm{~g} / \mathrm{d}$ against $0.69 \pm 0.11 \mathrm{~g} / \mathrm{d})$. Regarding the specific weight growth rate, its evolution was similar to that of the DWG. The recorded value was $4.71 \pm 0.22 \%$ / d for fish from fertilized ponds against $4.07 \pm 0.01 \% / \mathrm{d}$ for fish from unfertilized ponds. As for survival, of the order of $95 \%$, it was roughly identical in all breeding environments. These results suggest that the supply of fertilizer (droppings) contributes to the growth of fish by increasing primary production, an additional source of food.

Keywords: Organic fertilization, growth, survival, Oreochromis niloticus

\section{Introduction}

En Côte d'Ivoire, la production halieutique occupe une place importante dans l'économie nationale et constitue par conséquent, la principale source de protéine animale. La consommation annuelle par habitant de cette production est en moyenne de 15,9 kg (MIPRAH, 2014).Les besoins annuels en produits halieutiques et particulièrement en poisson sont compris entre 250000 et $300000 \mathrm{~T}$ contre une production locale avoisinant $80000 \mathrm{~T}$ (Yao et al., 2017). Face à ces besoins de plus en plus croissants dus à la démographie galopante, l'Etat ivoirien s'est engagé dans une politique de développement de l'aquaculture. Ce secteur d'activité avec une production de 3750 tonnes en 2014, malgré les nombreux investissements, ne représente que $5 \%$ de la production halieutique nationale (FAO, 2016). Cette situation est liée à plusieurs contraintes dont les faibles performances de croissance des espèces aquacultivables et l'aliment qui occupe entre 50 et $70 \%$ du coût de production (Adande et Fiogbé, 2015). Dès lors, l'utilisation de fertilisants organiques dans les étangs en vue d'optimiser les rendements de la production piscicole s'avère nécessaire. En effet, leur utilisation réduirait les coûts de production et améliorerait les performances de croissance par l'augmentation de la productivité primaire qui constitue une source d'aliment complémentaire pour les poissons (Adande et Fiogbé, 2015).

Dans la perspective d'une contribution au développement de la pisciculture, la présente étude se propose d'étudierl'effet de la fertilisation organique des étangs à partir de fiente (litière) de poulet préalablement composté sur la survie et la croissance des alevins de Oreochromis niloticus en phase de prégrossissement. 


\section{Matériel et méthodes \\ Site expérimental}

La présente étude a été réalisée dans une ferme piscicole située à Débrimou, dans le Département de Dabou (Sud-ouest de la Côte d'Ivoire). Dans cette ferme, se trouvent vingt-cinq (25) étangs de différentes dimensions dont certains abritent les cages et les happas. Tous ces étangs, disposés en parallèle, sont alimentés par une eau provenant d'une rivière à partir d'un canal de dérivation.

\section{Matériel}

\section{Matériel biologique}

Les expériences ont porté sur une espèce de Tilapia, Oreochromis niloticus (souche non déterminée). Les géniteurs d'un poids moyens compris entre 100 et $150 \mathrm{~g}$ ont servi à produire les alevins utilisés pour les tests de survie et de croissance.

\section{Matériel technique}

La production des larves a été réalisées dans des happas de dimension $3 \times 2 \times 1,5 \mathrm{~m}$ installés dans un étang piscicole de $400 \mathrm{~m}^{2}$. Les tests de survie et de croissance en phase de prégrossissement ont été conduits dans six (06) étangs de $200 \mathrm{~m}^{2}$. Durant toute l'expérimentation, l'oxygène dissous, le $\mathrm{pH}$ et la température de l'eau ont été mesurés respectivement à l'aide d'un oxymètre de type WTW OXI 330 et d'un pH-mètre (modèle WTW pH 90) couplé à un thermomètre. La taille et le poids des poissons ont été déterminés respectivement à l'aide d'un ichtyomètre et d'une balance électronique de type SARTORIUS et de précision $0,01 \mathrm{~g}$. Des épuisettes à toile moustiquaire, des seaux et des bassines en plastique ont servi à la pêche et au transport des poissons.

\section{Méthodes}

\section{Production des alevins}

Trois (3) happas ont été utilisés pour la reproduction des géniteurs. Ainsi, 16 géniteurs (4 mâles +12 femelles) ont été mis en reproduction dans chaque happa selon un sex ratio d'un mâle pour trois femelles. Durant la production des larves, les géniteurs ont été nourris deux fois par jour (entre $7 \mathrm{~h}$ et $8 \mathrm{~h}$ et entre $16 \mathrm{~h}$ et $17 \mathrm{~h}$ ) à l'aide d'un aliment granulé pour Tilapia, titrant $30 \%$ de protéines, à la ration journalière de $4 \%$ de la biomasse. Trois récoltes des larves ont été réalisées. La première est intervenue un mois après la mise en reproduction des géniteurs dans les happas. La seconde récolte a été effectuée 14 jours après la première. Enfin, la troisième récolte a aussi été réalisée 14 jours après la seconde. La récolte a consisté à pêcher à l'aide 
d'épuisettes, les larves qui ont été par la suite stockées dans d'autres happas prévus à cet effet.

\section{Constitution des lots et étude de la survie et de la croissance des alevins en prégrossissement}

Après les différentes récoltes, 24000 alevins de poids moyens initial compris entre $3,93 \pm 0,62 \mathrm{~g}$ et $3,97 \pm 0,31 \mathrm{~g}$ ont été sélectionnés et répartis équitablement dans six (6) étangs de $200 \mathrm{~m}^{2}$ à la densité de 20 alevins $/ \mathrm{m}^{2}$. Trois étang sont reçu une application d'engrais organique (fiente de poulet) à la dose $10 \mathrm{~kg} / \mathrm{m}^{2}$ par mois tandis que les trois autres pris comme témoins n'ont pas été fertilisés. Les alevins ont été nourris trois fois par jour à l'aide d'un aliment farineux titrant $35 \%$ de protéines à la ration journalière de $10 \%$ de la biomasse durant deux mois. Les paramètres physico-chimiques de l'eau ont été mesurés chaque jour, matin et soir (entre $7 \mathrm{~h}$ et $8 \mathrm{~h}$ matin et entre $16 \mathrm{~h}$ et $17 \mathrm{~h}$ le soir). Le suivi du poids et de la taille des poissons a été fait toutes les deux semaines à partir d'un échantillon de deux cent (200) alevins pêchés par étang. A la fin de l'essai, tous les étangs ont été vidés et le nombre d'alevins restant a été déterminé afin de calculer le taux de survie.

Les paramètres zootechniques suivis sont :

\section{Gain moyen quotidien (GMQ)}

GMQ $(\mathrm{g} / \mathrm{j})=($ Masse moyenne finale - Masse moyenne initiale $) /$ temps $(\mathrm{j})$

Taux de croissance spécifique linéaire(TCSL)

$\operatorname{TCSL}(\% / \mathrm{j})=[\ln ($ longueur moyenne finale $)-\ln ($ longueur moyenne initiale $)$ / Nombre de jours] x 100

\section{Taux de croissance spécifique pondérale (TCSP)}

$\operatorname{TCSP}(\% / \mathrm{j})=[\ln ($ poids moyen final $)-\ln ($ poids moyen initial $) /$ Nombre de jours] x 100

\section{Indice de consommation (IC)}

IC = Quantité d'aliment distribuée (g) / Gain de poids (g)

avec, Gain de poids $=$ Poids final - Poids initial

Taux de survie (TS)

TS $(\%)=($ Nombre final de poisson / Nombre initial de poisson $) \times 100$

\section{Analyses statistiques}

Les résultats ont été présentés sous forme de moyenne \pm écarts type entre réplicats. Les valeurs moyennes enregistrées sur les deux traitements (étangs fertilisés et étangs non fertilisés) ont été comparées par le test-t de 
Student. Les différences entre traitements ont été considérées significatives au seuil de $5 \%(p<0,05)$. Les analyses ont été effectuées à l'aide du programme STATISTICA 7.1.

\section{Résultats}

\section{Paramètres physico-chimiques}

Le tableau 1 présente les valeurs moyennes des paramètres physicochimiques de l'eau durant toute la période d'expérimentation. Ces paramètres n'ont pas significativement varié $(\mathrm{p}>0,05)$ d'un étang à un autre durant les deux mois de prégrossissement. Les limites de variation notées dans les étangs fertilisé sont été de 3,20 à $5,21 \mathrm{mg} / \mathrm{L}$ pour l'oxygène dissous, de 6,70 à 7,10 pour le $\mathrm{pH}$ et de 27,10 à $29,91^{\circ} \mathrm{C}$ pour la température. Quand aux limites de variations observées dans les étangs non fertilisés, elles ont été de 3,19 à 5,16 $\mathrm{mg} / \mathrm{L}$ pour l'oxygène dissous, de 6,68 à 7,02 pour le $\mathrm{pH}$ et de 27,50 à $29,80^{\circ} \mathrm{C}$ pour la température.

Tableau 1 : Valeurs moyennes des paramètres physico-chimiques de l'eau dans les étangs fertilisés et non fertilisés

\begin{tabular}{ccccc}
\hline Milieu & \multicolumn{4}{c}{ Paramètres physico-chimiques } \\
\cline { 2 - 5 } d'élevage & & $\mathbf{O}_{\mathbf{2}}(\mathbf{m g} / \mathbf{l})$ & $\mathbf{p H}$ & Temp $\left({ }^{\circ} \mathbf{C}\right)$ \\
\hline \multirow{2}{*}{ Etangs } & Min & 3,20 & 6,70 & 27,10 \\
fertilisés & Max & 5,21 & 7,10 & 29,91 \\
& Moy & $\mathbf{4 , 0 2}$ & $\mathbf{6 , 7 2}$ & $\mathbf{2 9 , 1 0}$ \\
& E-type & 1,01 & 0,01 & 0,03 \\
\hline \multirow{2}{*}{ Etangs non } & Min & 3,19 & 6,68 & 27,50 \\
fertilisés & Max & 5,16 & 7,02 & 29,80 \\
& Moy & $\mathbf{4 , 1 1}$ & $\mathbf{6 , 6 9}$ & $\mathbf{2 9 , 0 1}$ \\
& E-type & 0,91 & 0,03 & 0,08 \\
\hline
\end{tabular}

(Temp : Température ; $\mathrm{O}_{2}$ : Taux d'oxygène dissous ; $\mathrm{pH}$ : potentiel d'Hydrogène ; Min : valeur minimale ; Max : valeur maximale ; Moy : moyenne entre réplicats et E-type : écarttype entre réplicats).

\section{Performances zootechniques}

Les paramètres zootechniques des poissons après deux mois de prégrossissement, sont présentés dans le tableau 2. A la fin de l'expérience, le gain moyen quotidien (GMQ) a été plus important au niveau des poissons élevés dans les étangs fertilisés que celui enregistré au niveau des étangs non fertilisés $(1,04 \pm 0,17 \mathrm{~g} / \mathrm{j}$ contre $0,75 \pm 0,11 \mathrm{~g} / \mathrm{j})$. En ce qui concerne le taux de croissance spécifique pondérale, il présente la même tendance que celle du GMQ. La valeur enregistrée est de 4,71 $\pm 0,22 \% / j$ pour les poissons des étangs fertilisés et de $4,07 \pm 0,01 \% / \mathrm{j}$ pour les poissons des étangs non fertilisés. La comparaison statistique de ces paramètres montre une différence significative $(\mathrm{p}<0,05)$ en faveur des poissons élevés dans les étangs fertilisés. Pour le taux de croissance spécifique en longueur, aucune différence significative n'a été 
observée $(p>0,05)$ au niveau des deux types de milieu d'élevage (étangs fertilisés et étangs non fertilisé). Quant aux valeurs des indices de consommation, elles ont été de 2,10 $\pm 0,30$ (étangs fertilisés) et de $3,8 \pm 0,81$ (étangs non fertilisés). La comparaison statistique montre une différence significative $(\mathrm{p}<0,05)$ en faveur des poissons des étangs non fertilisés.

Les taux de survie obtenus ont été élevés et sensiblement identiques dans les deux types de milieux (95,52\% dans les étangs fertilisés et 94,91\% dans les étangs non fertilisés).

Tableau 2 : Paramètres zootechniques de Oreochromis niloticus enregistrés après deux mois de prégrossissement en étangs

\begin{tabular}{ccc}
\hline \multirow{2}{*}{ Paramètres } & \multicolumn{2}{c}{ Milieux d'élevage } \\
\cline { 2 - 3 } & Etangs fertilisés & Etangs non fertilisés \\
\hline $\operatorname{Li}(\mathrm{cm})$ & $5,82 \pm 0,21^{\mathrm{a}}$ & $5,86 \pm 0,14^{\mathrm{a}}$ \\
$\mathrm{Lf}(\mathrm{cm})$ & $14,04 \pm 0,16^{\mathrm{a}}$ & $13,50 \pm 0,02^{\mathrm{b}}$ \\
$\mathrm{Pi}(\mathrm{g})$ & $3,93 \pm 0,62^{\mathrm{a}}$ & $3,97 \pm 0,31^{\mathrm{a}}$ \\
$\mathrm{Pf}(\mathrm{g})$ & $66,61 \pm 1,61^{\mathrm{a}}$ & $45,89 \pm 1,20^{\mathrm{b}}$ \\
$\mathrm{GMQ}(\mathrm{g} / \mathrm{j})$ & $1,04 \pm 0,17^{\mathrm{a}}$ & $0,69 \pm 0,11^{\mathrm{b}}$ \\
$\mathrm{TCSL}(\% / \mathrm{j})$ & $1,46 \pm 0,01^{\mathrm{a}}$ & $1,39 \pm 0,21^{\mathrm{a}}$ \\
TCSP $(\% / \mathrm{j})$ & $4,71 \pm 0,22^{\mathrm{a}}$ & $4,07 \pm 0,01^{\mathrm{b}}$ \\
$\mathrm{IC}$ & $2,10 \pm 0,30^{\mathrm{b}}$ & $3,80 \pm 0,81^{\mathrm{a}}$ \\
$\mathrm{TS}(\%)$ & $95,52 \pm 0,20$ & $94,91 \pm 0,10$ \\
\hline
\end{tabular}

NB: les valeurs de la même ligne, exprimées en moyenne \pm écart type, indexées des mêmes lettres alphabétiques ne sont pas statistiquement différentes $(p>0,05) . \mathrm{Li}=$ Longueur initiale $; \mathrm{Lf}=$ Longueur finale $; \mathrm{Pi}=$ Poids initial $; \mathrm{Pf}=$ Poids final $; \mathrm{GMQ}=$ Gain moyen quotidien $;$ TCSP $=$ Taux de croissance spécifique en poids $;$ TCSL $=$ Taux de croissance spécifique en longueur ; IC = Indice de consommation ; TS = Taux de survie.

\section{Discussion}

Dans la présente étude, les paramètres physico-chimiques de l'eau enregistrés dans les étangs (fertilisés et non fertilisés) se situent dans les limites recommandées pour l'élevage des tilapias. En effet, selon Stickney (1986) et Faye et al. (2018), les valeurs de pH les plus favorables pour la culture des tilapias sont comprises entre 6,5 et 8,5. Mims et Shelton (2015) indiquent que Oreochromis niloticus peut vivre dans des zones à pH compris entre 5 et 11. En ce qui concerne la température, Dabbadié et al. (2006) affirment que le tilapia est thermophile et préfère les températures comprises entre 14 et $35^{\circ} \mathrm{C}$. Pour Faye et al. (2018), une température de $27,7 \pm 1,24{ }^{\circ} \mathrm{C}$ est favorable à l'élevage des tilapias ; ce qui est en accord avec nos résultats. Concernant l'oxygène dissous, la valeur moyenne obtenue $(4,02 \pm 1,01 \mathrm{mg} / \mathrm{L})$ est au-dessus de la valeur seuil en phase de croissance $(2,3 \mathrm{mg} / \mathrm{L})$ rapportée par Ross (2000). Ces paramètres n'auraient donc pas une influence sur la survie et la croissance des alevins de Oreochromis niloticus dans la présente étude. Les mortalités relevées pourraient être d'origine naturelle ou être 
provoquées par les chocs reçus par les alevins lors des opérations d'échantillonnage.

Les performances de croissance observées au niveau des poissons élevés en étangs fertilisés ont été plus importantes que celles enregistrées au niveau des étangs non fertilisés. Cette situation pourrait s'expliquer par l'apport de fertilisant organique (fientes de poulet) qui a favorisé le développement du plancton, source de nourriture naturelle pour les poissons. En effet, les fientes sont adaptées à l'élevage des tilapias. Elles jouent non seulement le rôle d'engrais pour le développement du plancton, mais aussi, représentent une source immédiate de nourriture car le tilapia peut se nourrir de détritus (Mikolasek, 2009). De plus, Barbe et al. (2010) et FAO (2015) ont montré que la rentabilité d'un plan d'eau dépend de la richesse du milieu en plancton qui elle-même est fonction des apports de fertilisants organiques. Selon Adande et Fiogbé (2015), les fertilisants organiques représentent un vivier important pour le développement de la pisciculture car ces derniers ont l'aptitude de libérer des sels nutritifs indispensables dans l'eau pour la production primaire. Ben et al. (2014) ont aussi montré que les pertes d'élevage des fermes induisent le développement des algues, du zooplancton et des macroinvertébrés, qui sont des sources nutritives supplémentaires favorables à la croissance des poissons. Par ailleurs, Hecth (2013) indique que la fertilisation organique des étangs assure une bonne production de zooplancton, source de nourriture pour les poissons. Durant cette phase de production, en étangs fertilisés comme non fertilisés, les valeurs du GMQ obtenues sont supérieures à celle obtenue par Amon et al. (20013) en bacs en béton $(0,69$ à $1,04 \mathrm{~g} / \mathrm{j}$ contre $0,60 \mathrm{~g} / \mathrm{j})$. Cette différence pourrait s'expliquer par les structures d'élevage utilisées. En effet, selon Bombéo et al. (2002), les étangs offrent la possibilité d'un développement plus important de la productivité naturelle qu'en bassins en béton.

\section{Conclusion}

La présente étude avait pour but de voir l'effet de la fertilisation organique des étangs à partir des fientes de poulets sur la survie et la croissance des alevins de Oreochromis niloticus. Il apparait clairement que l'apport de fertilisant (fiente) contribue à la croissance des poissons par l'augmentation de la production primaire, source supplémentaire de nourriture. Cependant, il serait intéressant dans des études ultérieures, de quantifier cette production primaire en fonction des types de fertilisants afin de choisir le meilleur pour une production piscicole optimale.

Conflits d'intérêts : Les auteurs déclarent n'avoir aucun conflit d'intérêt. 


\section{References :}

1. Adande R. \& Fiogbe E. D.(2015). Utilisation des fertilisants organiques d'origine animale et végétale pour le développement de la pisciculture dans les étangs : Synthèse bibliographique. International Journal of Multidisciplinary Research and Development, 2 (12): 281287.

2. Amon Y. N., Yao K., Atsé B. C. \& Ouattara M. (2013). Survie et croissance des juvéniles hybrides issus du croisement intergénérique Oreochromis niloticus (linnaeus, 1758) et Sarotherodon melanotheron (Rüppel, 1852) en milieu lagunaire. International Journal of Biological and Chemical Sciences, 3 (7): 1069-1077.

3. Balarin J. D. \& Haller R.D. (1982). The intensive culture of tilapia in tanks, raceways and cages. In: J.F.Muir and Roberts RJ. (Eds.), Recent Advances in Aquaculture, Vol. 1. CroomHelm, London.

4. Barbe J., Schlumberger O. \& Bouretz N. (2000).Evaluation de la production piscicole potentielle des étangs. Ingénieries-EAT : 49-62.

5. Ben M., Chahlaoui A., Rour E. \& Chahboune M.(2014). Diversité taxonomique et structure de la macrofaune benthique des eaux superficielles de l'oued khoumane River. Moulay idriss Zerhoun, Maroc J Mater Environ Sci., 1(5):183-198.

6. Bombéo R. F., Fermin A. C. \& Tanfermin J. D. (2002). Nursery rearing of the Asian catfish, Clarias macrocephalus (Günther) at different stocking densities in cages suspended in tanks and ponds. Aquaculture Research 33 (13): 1031-1036.

7. Dabbadie L., Lazard J. \& Oswald M.(2006). La pisciculture. In Greet et al. Mémento de l'agronome, Montpellier, France : 1571-1615.

8. FAO (2015). Engrais et fertilisant : système d'information sur les ressources alimentaires et d'engrais en aquaculture:

http//www.fao.org/fishery/affris/profil-des-speces/niletilapia/engraiset-fertilisant/fr/ $1 / 2$.

9. FAO (2016). La situation mondiale des pêches et de l'aquaculture. Contribution à la sécurité alimentaire et à la nutrition de tous. Rome, Italie, 105p.

10. Faye E., Sarr S. M., Touré M. A., Gueye S. \& Gueye M. (2018). Effets de la densité de stockage sur la croissance des alevins de Tilapia (Oreochromis niloticus L.) en cages fixes dans le Lac de Guiers, Sénégal. AfriqueSCIENCE 14 (3): 378-390.

11. Hecht T. (2013). A review of on-farm feed management practices for North African catfish (Clarias gariepinus) in sub-Saharan Africa. In M.R. Hasan and M.B. New, eds. On-farm feeding and feed management in aquaculture. FAO Fisheries and Aquaculture Technical Paper, Rome, FAO 2013, 583:463-479. 
12. Mikolasek O., Barlet B., Chia E., Pouomogne V. \& Tabi M. T. E. (2009). Développement de la petite pisciculture marchande auCameroun : la recherche-action en partenariat. Cah. Agric, vol. 18, no. 2-3: 270-276.

13. Mims S. D. \& Shelton W. L. (2015). Propagation and Early Culture Techniques. In Steven D. Mims and William L. Shelton (éds): Paddlefish Aquaculture. John Wiley \& Sons, Hoboken, New Jersey: 77-127.

14. MIPRAH (2014). Plan stratégique de développement de l'élevage, de la pêche et de l'aquaculture en Côte d'Ivoire (PSDEPA 2014-2020). Tome I : Diagnostic - Stratégie de développement - Orientations stratégiques, $102 \mathrm{p}$.

15. Ross L. G. (2000). Environmental physiology and energetic. In: Bevereridge, M. C. M. et McAndrew, B. J. (Eds.). Tilapias: Biology and Exploitation. Dordrecht, Netherlands: Kluwer Academic Publisher, Fish and Fisheries series 25: 89-128.

16. Stickney R. R. (1986). Culture of nonsalmonid freshwater fishes, Boca Roton, USA: CRC Press, 201p.

17. Yao A. H., Koumi A. R., Atsé B. C. \& Kouamélan E. P. (2017). Etat des connaissances sur la pisciculture en Côte d'Ivoire. Agronomie Africaine 29 (3) : 227-244. 